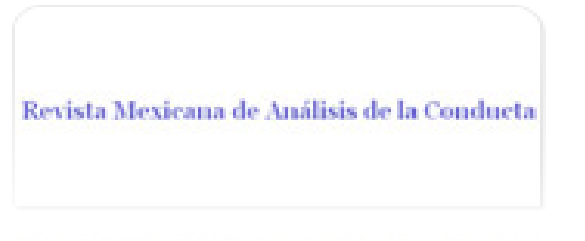

Revista Mexicana de Análisis de la Conducta ISSN: 0185-4534

editora@rmac-mx.org

Sociedad Mexicana de Análisis de la Conducta México

LÓPEZ-ROMERO, LUIS; ALVARADO, ANGÉLICA; VILA, JAVIER

LA REGLA DEL PESO TEMPORAL Y LA RECUPERACIÓN ESPONTÁNEA EN HUMANOS

Revista Mexicana de Análisis de la Conducta, vol. 36, núm. 1, junio, 2010, pp. 85-96

Sociedad Mexicana de Análisis de la Conducta

Guadalajara, México

Disponible en: http://www.redalyc.org/articulo.oa?id=59313134006

Cómo citar el artículo

Número completo

- Más información del artículo

Página de la revista en redalyc.org

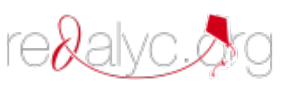

Sistema de Información Científica

Red de Revistas Científicas de América Latina, el Caribe, España y Portugal Proyecto académico sin fines de lucro, desarrollado bajo la iniciativa de acceso abierto 


\title{
LA REGLA DEL PESO TEMPORAL Y LA RECUPERACIÓN ESPONTÁNEA EN HUMANOS
}

\author{
TEMPORAL WEIGHTING RULE AND SPONTANEOUS RECOVERY IN \\ HUMANS
}

\author{
LUIS LÓPEZ-ROMERO, ANGÉLICA ALVARADO \\ Y JAVIER VILA* \\ DIV. DE INVESTIGACIÓN Y POSGRADO \\ FACULTAD DE ESTUDIOS SUPERIORES IZTACALA \\ UNIVERSIDAD NACIONAL AUTÓNOMA DE MÉXICO
}

\section{Resumen}

El objetivo del presente trabajo fue el de proponer un modelo capaz de explicar la recuperación espontánea observada en el aprendizaje instrumental humano. Se presentan un experimento que demuestra que la información aprendida es integrada con el paso del tiempo en el resurgimiento del material olvidado. Se sugiere así la aplicación al estudio de la recuperación espontánea con humanos del modelo de la regla del peso temporal derivado de estudios de alimentación en animales (Devenport, 1998). Este modelo propone que las experiencias son recordadas de acuerdo a su distancia temporal relativa y su valor subjetivo. Así en base a la manipulación del parámetro temporal de distancia relativa y el del valor subjetivo de cada experiencia, se comprobó en humanos que la recuperación espontánea es una función del valor del intervalo de retención sólo cuando el primer aprendizaje tiene un mayor valor subjetivo. Los resultados muestran una integración de las experiencias aprendidas con el paso del tiempo en función del valor del intervalo de retención. También sugieren que es posible predecir y observar el desarrollo de la recuperación espontánea en tareas de aprendizaje instrumental con humanos con base a la regla del peso temporal.

Palabras clave; tiempo, memoria, recuperación espontánea, regla del peso temporal, humanos.

* La presente investigación fue apoyada financieramente por los proyectos DGAPA-UNAM (IN302910-2) y CONACYT (52525H) y forma parte de la tesis doctoral del primer autor. El diseño y conducción, del estudio estuvo a cargo del primer autor, el análisis de los datos estuvo a cargo del segundo autor y la discusión fue responsabilidad del tercer autor. La correspondencia relacionada a presente articulo puede dirigirse a Luis Jesús Lopez Romero, e-mail. luisjir@correo.unam.mxy/oAngélica SerenaAlvarado García Posgrado, Facultad de Estudios Superiores Iztacala, UNAM AP 314, Tlalnepantla, Edo. Mex, 54096. 
Abstract

The present study was designed to propose a model that may explain human spontaneous recovery. In the present experiment we explored whether previously learned information would be integrated by the passage of time and hence produce reappearance of the forgotten material. Accordingly, we suggest that the Temporal Weighting Rule, derived from animal foraging studies (Devenport, 1998), may be applied to the study of human spontaneous recovery. Temporal Weighting Rule assumes that experiences are remembered depending on the relative temporal distance and subjective value. Manipulations of the temporal and subjective values in humans confirm one of the main predictions of the model. Spontaneous recovery is a function of the retention interval value only when the initial learning has grater subjective value. Results showed that spontaneous recovery is closely modeled by the Temporal Weighting Rule model and suggest also that it can predict the development of spontaneous recovery in human instrumental learning tasks.

Key words: time, memory, spontaneous recovery, temporal weighting rule, humans.

Pavlov (1927) observó originalmente la recuperación espontánea (RE) como un fenómeno que ocurre posteriormente a la extinción cuando se presenta la reaparición de una respuesta extinguida después de un intervalo de retención (IR). En virtud de que la $R E$ se ha observado en el condicionamiento clásico e instrumental, tanto en aprendizaje animal (Ellson, 1938; Stollhoff, Menzel, \& Eisenhardt, 2005), como en aprendizaje humano (Postman, Stark, \& Fraser, 1968; Vila, Romero, \& Rosas, 2002) se le ha considerado un fenómeno robusto de aprendizaje.

Actualmente las explicaciones de la RE se han centrado en algunas de las siguientes consideraciones teóricas: el debilitamiento de asociaciones inhibitorias y recuperación de las excitatorias con el paso del tiempo (Pavlov, 1927), la ausencia de consolidación de la memoria de extinción que permite el resurgimiento de la respuesta (Spear, 1973) y la interferencia entre memorias contradictorias que se disipa con un cambio de contexto temporal (Bouton, 1993).

La teoría más aceptada y conocida de la RE es la teoría de la interferencia propuesta por Bouton (1993) en la que la recuperación espontánea se considera como un efecto producido por el cambio de contexto temporal.

De acuerdo con este punto de vista la extinción es vista como una fase de interferencia retroactiva dependiente del contexto. Así un cambio en el contexto de extinción producirá la reaparición de la respuesta extinguida, produciéndose un efecto de renovación contextual (Bouton \& King, 1983). 
Bouton supone que el paso del tiempo puede actuar como un cambio de contexto físico o interno, de esta manera en el caso de la RE después de que la adquisición y la extinción han ocurrido en el mismo contexto como consecuencia del paso del tiempo durante el IR, el contexto cambiará produciendo un efecto de renovación contextual.

Se puede suponer así que en la RE a medida que el tiempo pasa el contexto donde se llevó a cabo la extinción va cambiando cada vez más favoreciendo la reactivación de la primera información adquirida generándose así un cambio "recencia a primacía" en el que el aprendizaje más reciente es substituido por un aprendizaje inicial (Knoedler, Hellwig, \& Neath, 1999). En donde el paso del tiempo producirá un mayor cambio del contexto y por tanto una mayor recuperación. Con lo cual se puede suponer que la magnitud de la RE estará en función de la duración del IR.

Al presente esta predicción ha sido difícil de comprobar ya que en la mayoría de los estudios con humanos y animales la RE obtenida ha sido parcial, por lo que el cambio recencia-primacía producido por el incremento del valor del IR ha sido difícil de observar (Stout, \& Miller, 2005; Thomas, Larsen, \& Ayres, 2003; Vila et al., 2002;). Estos hallazgos han llevado a algunos autores a proponer alternativamente que la información aprendida durante las fases de adquisición y extinción es integrada con el paso del tiempo y que la RE es la manifestación de la integración de lo aprendido en ambas fases de entrenamiento (Alvarado, Jara, Vila \& Rosas, 2006; López-Romero et al., 2010).

Por lo que un cambio recencia-primacía no siempre ocurrirá como función del valor del IR. Por ejemplo, Romero, Vila y Overmier (2007) han demostrado que la RE puede ser una función de otras variables que modifican el valor de cada experiencia aprendida. En este trabajo se estudió el efecto del IR en una tarea de interferencia con dos consecuencias en humanos. Así, empleando un tarea de discriminación condicional inversa en humanos con dos consecuencias diferentes observaron una interacción entre el IR y el tipo de consecuencia en el cual la magnitud de la RE fue una función de si las consecuencias diferenciales ocurrían en la primera o segunda fase del entrenamiento. Lo anterior sugiere que consecuencias diferentes pueden funcionar como señales capaces de producir una mayor o menor RE sin importar la longitud del IR.

Por otro lado un punto de vista teórico reciente derivado de estudios de forrajeo en animales considera también que la RE es una integración de lo aprendido en fases previas de entrenamiento anteriores a un IR (Devenport, 1998; Devenport \& Devenport, 1994). 
Esta aproximación teórica es conocida en la literatura como la regla del peso temporal (RPT), y propone que cuando se aprenden dos experiencias sucesivamente aquellas que son más recientes tendrán un mayor peso si se hace una prueba inmediata, pero que esta valoración cambiará con el paso del tiempo, en una prueba posterior dando lugar a la ocurrencia de la recuperación espontánea. Según Devenport (1998) lo que regula la transición en la elección entre dos alternativas de respuesta es el paso del tiempo, de esta manera la RE refleja la elección de una opción que en promedio fue la de mayor valor subjetivo durante el entrenamiento, ya que los valores de ambas experiencias tienden a ser iguales con el paso del tiempo.

De acuerdo con la RPT la RE se explica al considerar que los organismos usan un promedio dinámico que toma en cuenta el valor subjetivo y el valor temporal de las memorias de cada experiencia aprendida. Así, la memoria de las experiencias recientes tiene un mayor peso en la ejecución inmediatamente después de la experiencia (recencia) pero este valor se reduce con el paso del tiempo. Es decir, si en la primera fase se presentan ensayos reforzados y en la segunda fase se presentan ensayos no reforzados para una misma clave, se observará un efecto de recencia de extinción si la prueba se realiza inmediatamente después de la segunda fase, pero una RE de la memoria de la primera fase si la prueba se realiza después de un intervalo de retención; efecto que se observa también en una situación en donde el sujeto tiene que elegir entre dos claves que han sido reforzadas y extinguidas en diferente orden (Devenport, 1998). Una predicción derivada de esta idea supone que la RE con IR mayores a cero mostrará indiferencia entre las dos experiencias cuando las dos experiencias tengan el mismo valor subjetivo y distancia relativa.

Esta idea ha sido comprobada recientemente en la recuperación espontánea con humanos empleando preparaciones de aprendizaje causal (Alvarado et al., 2006) y de discriminación condicional en condicionamiento instrumental (López-Romero et al., 2010). En estos estudios la RE no es una función de la longitud del IR, sino que al disiparse la recencia de la primera experiencia con el paso del tiempo y aumentar el valor del IR se observó una RE que muestra indiferencia por ambas experiencias en pruebas realizadas con varios valores del IR y no se observó un cambio de recencia a primacía tal y como lo predice la teoría de la interferencia de Bouton.

Sin embargo, la RPT puede predecir un cambio recencia-primacía en la que la RE es una función del valor del IR sólo cuando la primera experiencia tiene un valor subjetivo mayor que la segunda experiencia tal y como ocurre en la extinción debido al reforzamiento sólo durante la primera fase.

Así después del IR si bien ambas experiencias son iguales en su distancia relativa, la primera experiencia tendrá un mayor peso al ser considerada en el momento de prueba debido a su mayor valor subjetivo. 
Este supuesto parece haber sido comprobado recientemente en un estudio con humanos empleando una tarea de aprendizaje predictivo en la que los participantes aprendían a predecir la ocurrencia de una consecuencia en presencia de una clave determinada (Stout et al., 2005). Sin embargo, estos autores emplearon solo dos valores del IR (0 y $48 \mathrm{hr})$ posteriormente a la extinción y por tanto no observaron una RE completa.

Por ello en el presente trabajo se pretende comprobar, con participantes humanos y empleando una tarea de aprendizaje instrumental, el supuesto del cambio recencia-primacía con varios valores del IR, cuando el valor subjetivo de la primera experiencia es subjetivamente mayor al de la segunda experiencia en un entrenamiento adquisición- extinción. De acuerdo con los supuestos de la RPT propuesta por Devenport (1998) la RE será mayor al aumentar el valor del IR cuando la primera experiencia aprendida sea de un valor subjetivo mayor al de la segunda experiencia produciendo un cambio recencia-primacía en el que la recuperación de lo aprendido sea una función del valor del IR. De esta manera inicialmente en valores cortos del IR la memoria de la segunda experiencia será más reciente y por tanto tendrá un mayor peso, produciendo poca RE. Sin embargo al aumentar el valor del IR la recencia se desvanecerá y la distancia temporal relativa será similar para ambas experiencias, por lo que la memoria de la primera fase de adquisición será la de la experiencia con un mayor valor subjetivo que tendrá entonces mayor peso y por tanto se producirá una mayor RE. Para estudiar esta predicción se diseñó un experimento en el que los participantes aprendieron a resolver una tarea que se presentaba en la pantalla de una computadora tomando en cuenta el diseño que utilizó Devenport (1998) para someter a prueba una de las predicciones de la RPT según la cual se puede predecir una curva de recuperación de la respuesta en base a diferentes valores del intervalo entrenamiento-prueba.

Método

\section{Participantes}

Colaboraron voluntariamente 48 estudiantes universitarios de la FESIIztacala, de la Universidad Nacional Autónoma de México, quienes fueron asignados aleatoriamente a cuatro grupos experimentales. Los estudiantes tenían entre 17 y 21 años de edad y no contaban con ninguna experiencia en la tarea experimental. Su participación fue solicitada de acuerdo a las normas éticas de investigación con humanos a través del consentimiento informado. 
Aparatos y situación experimental

El experimento se llevó a cabo de manera individual en un cubículo de 2x2 $\mathrm{m}$. Se empleó una computadora personal para presentar la tarea y se utilizó el programa informático Super Lab Pro para Windows v 4.0.2 (Cedrus Co., San Pedro, California, EEUU) para realizar los experimentos, presentar los estímulos y registrar las respuestas de los participantes.

\section{Tarea experimental.}

La tarea experimental consistió en la elección de uno de dos estímulos que cambiaban en su valor temporal entre entrenamiento y prueba. Se presentó en la pantalla de un monitor de computadora una situación ficticia de un juego de cartas, donde se ganaban puntos bajo un programa de intervalo variable $5 \mathrm{~s}$ respondiendo en una de dos maquinas dispensadoras de cartas $(A=$ Dispensador Azul; $\mathrm{B}=$ Dispensador Amarillo) que se presentaban sucesivamente y de manera contrabalanceada. En cada fase sólo uno de los dispensadores permitía ganar puntos mientras que el otro dispensador se encontraba en extinción. Al finalizar el entrenamiento se presentó una fase de prueba con una duración de 1 min donde los participantes tuvieron que elegir entre las dos máquinas dispensadoras que se presentaron simultáneamente durante cuatro bloques de $15 \mathrm{~s}$.

\section{Procedimiento}

Los participantes se asignaron aleatoriamente a cada uno de los cuatro grupos experimentales $(n=12)$. Se les pidió que se sentaran frente al monitor de la computadora y que leyeran las instrucciones, la sesión experimental para cada grupo dio inicio de acuerdo al diseño correspondiente. En las fases de entrenamiento se presentaron 12 ensayos respuesta-consecuencia. Para todos los grupos el experimento constó de dos fases de entrenamiento y una fase de prueba (ver Tabla 1).

Tabla 1.

Descripción de las fases de entrenamiento e intervalos entrenamiento-prueba para los cuatro grupos de participantes.

\begin{tabular}{|c|c|c|c|c|}
\hline Grupo & Fase 1 & Fase 2 & Intervalo & Prueba \\
\hline $\mathrm{A}>\mathrm{B} 0 \mathrm{hr}$ & & & $0 \mathrm{hr}$ & \\
\hline $\mathrm{A}>\mathrm{B} 0.5 \mathrm{hr}$ & (6) $\mathrm{A}+++$ & (6) A - & $0.5 \mathrm{hr}$ & \\
\hline $\mathrm{A}>\mathrm{B} 1 \mathrm{hr}$ & (6) B- & (6) $\mathrm{B}+$ & $1 \mathrm{hr}$ & \\
\hline $\mathrm{A}>\mathrm{B} 24 \mathrm{hr}$ & & & $24 \mathrm{hr}$ & \\
\hline
\end{tabular}

Nota: Los nombres de los grupos corresponden a la magnitud de la consecuencia y al valor del intervalo entrenamiento-prueba, los valores encerrados entre paréntesis corresponden al número de ensayos, las letras en las celdas de las fases indican: A=Dispensador Azul ; B=Dispensador Amarillo y los signos $(+)=$ Reforzamiento y $(-)=$ Extinción 
El grupo A>B 0 hr recibió la Fase 1, Fase 2, y Prueba con las opciones A y B en la misma sesión. Los grupos A>B .5 hr, A>B $1 \mathrm{hr}$ y A>B $24 \mathrm{hr}$ recibieron las Fases 1 y 2 en la misma sesión y la prueba de elección se realizó después de un IR de.5 hr, $1 \mathrm{hr}$ y $24 \mathrm{hr}$. después, respectivamente. Durante las fases de entrenamiento la posición de los dispensadores de cartas se contrabalanceó mientras que en la Fase de Prueba los dispensadores aparecían simultáneamente y los bloques se presentaron al azar.

En grupo A>B 0 hr una vez que terminaba la segunda fase se les pidió a los participantes que salieran del cubículo a buscar al experimentador e inmediatamente después se les indicó que regresaran a terminar la tarea, mientras que en los grupos restantes cuando salieron del cubículo se les pidió que regresaran después del intervalo correspondiente sin que se les haya pedido que hicieran alguna actividad en particular. La magnitud de reforzamiento para A y B se programó de manera que durante la Fase 1 para $A$ se otorgaban 20 puntos en cada ensayo y para $B$ cero puntos, mientras que en la Fase 2 para A se otorgaban cero puntos mientras que para B se recibían cuatro puntos por ensayo.

Las variables a medir y analizar durante la prueba fueron la diferencia promedio en la elección A - B, que se calculó en base al promedio de las diferencias en la elección a las opciones de respuesta y la tasa local de respuestas que fue calculada dividiendo el tiempo de permanencia en una opción ( $t A$ ó tB) entre el tiempo total para ambas opciones ( $\mathrm{t} A+t B)$.

\section{Resultados}

En general los resultados mostraron que la RE obtenida es una función del valor del IR. La Figura 1 muestra el promedio de las diferencias en la elección de las opciones A y B. Como se puede observar, existe preferencia por la opción B en el grupo A>B $0 \mathrm{hr}$ con un valor de la diferencia promedio de -5 . Existe indiferencia en la elección del grupo $A>B .5 \mathrm{hr}$ con un valor en la diferencia promedio de 2 y preferencia por $A$ en los grupos $A>B$ 1h y $A>B 24 h$ con un valor de la diferencia promedio de $5 \mathrm{y}$ un valor de la diferencia promedio cercano a 10. Las diferencias A-B entre los cuatro grupos fueron analizadas mediante un ANOVA en el que observó una diferencia significativa $F(3,44)=$ $33.770, p<.00$. Para comprobar éstas diferencias un análisis post hoc utilizando la prueba LSD reveló que los grupos A>B $0 \mathrm{hr}, A>B$.5h, A>B $1 \mathrm{hr}$ y A>B 24 hr difirieron entre si $(p<.05)$. 


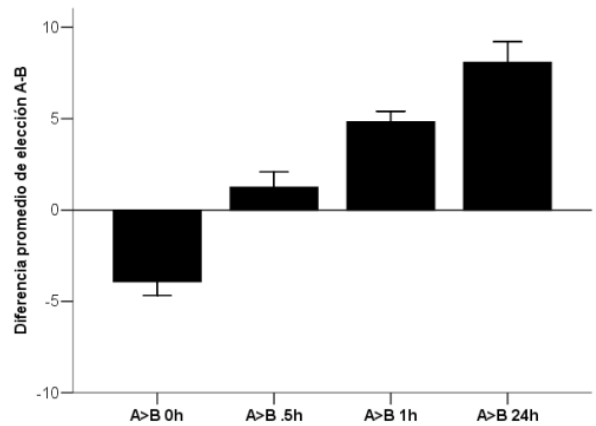

Figura 1.Diferencia promedio en la elección entre $A$ y $B$ en donde una puntuación positiva indica preferencia por la opción $A$, una puntuación negativa indica preferencia por la opción B y una puntuación cercana a 0 indica indiferencia en la elección, para cada uno de los grupos $(n=12)$ durante la prueba de elección.

En la Figura 2 se muestra la tasa local de respuesta por grupo que representa el tiempo que los participantes permanecían en cada dispensador durante la prueba. La tasa local fue calculada dividiendo el tiempo de permanencia en una opción ( $t A$ o tB) entre el tiempo total para ambas opciones $(t A+t B)$. Un ANOVA 4 (Grupo) x 2 (Permanencia A ó B) mostró efectos principales para permanencia $F(1,88)=39.171, p<0.0$, y un interacción significativa $F(3,88)=$ $22.595, p<0.0$, y ningún otro efecto fue significativo $p>.05$. Para analizar los efectos de interacción se realizaron comparaciones de la Tasa Local de Respuesta entre A y B con la prueba t de Student para cada grupo, en las que se encontraron diferencias en el grupo A>B $0 \mathrm{hr}(\mathrm{t}(88)=-3.469, p<.001$, que sugiere que los participantes permanecían más tiempo en $B$, mientras que en los grupos restantes ( $A>B .5 \mathrm{hr}, A>B 1 \mathrm{hr}$, y $A>B 24 \mathrm{hr}$ ) permanecían más tiempo en $\mathrm{A}, t(88)=3.019, p<.003 ; t(88)=-5.246, p<0.0$ y $t(88)=7.661, p<$ 0.0 , respectivamente. Lo que sugiere que al aumentar el IR el tiempo de permanencia en B disminuía mientras que la permanencia en A aumentaba.

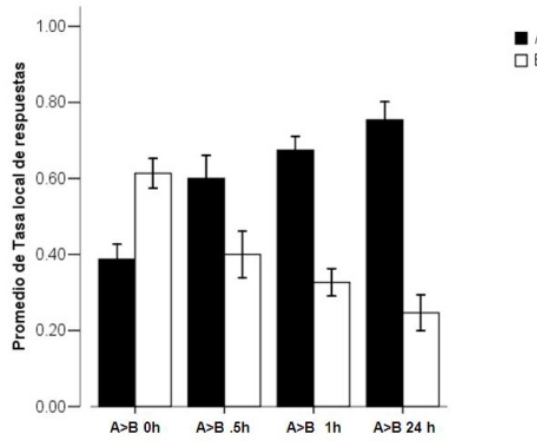

Figura 2.Promedio de tasa local de respuestas, calculada como el tiempo de permanencia en cada opción entre el tiempo total de prueba, para cada grupo $(n=12)$ durante la prueba de elección en donde las letras representan cada una de las opciones de respuesta $(A=$ Máquina $A z u l$ y $B=$ Máquina amarilla $)$. 
De acuerdo con estos resultados a medida que el valor del IR aumenta a valores mayores a $0 \mathrm{~h}$, la preferencia por A aumenta gradualmente, ya que fue la experiencia con mayor valor relativo durante la primera fase $(\mathrm{A}+++)$, mientras que la preferencia por $B$ disminuye desde una preferencia inicial a las $0 \mathrm{hr}$. Estos datos demuestran un efecto de cambio recencia-primacía gradual dependiente del valor del IR.

\section{Discusión}

Después de un entrenamiento en una tarea instrumental con dos fases adquisición-extinción donde los participantes aprendieron a responder a dos opciones de respuesta $A$ y $B$ que se diferenciaban en su valor subjetivo (magnitud de reforzamiento $A+++/ A-y ~ B-/ B+$ ) y en su valor de recencia entre el último ensayo de entrenamiento y una prueba de elección ( 0 vs.5,1 y 24 h), se observan diferentes efectos: a) cuando se realiza una prueba inmediata se observa preferencia por $\mathrm{B}$, que es la opción reforzada mas recientemente, aunque de menor magnitud en la consecuencia, b) cuando la prueba se realiza $.5,1$ y $24 \mathrm{hr}$ después del entrenamiento se observa una preferencia gradual hacia $A$, que es la opción aprendida inicialmente pero con una consecuencia de mayor magnitud.

Esta preferencia es proporcional al incremento del valor del intervalo entrenamiento-prueba y ocurre cuando del valor relativo de la primera experiencia $(\mathrm{A}+++)$ es mayor. Este efecto recencia-primacía se desarrolla gradualmente al aumentar el valor del IR y no de manera abrupta como lo proponen Stout et al., (2005). Sin embargo, cuando el valor de ambas experiencias es similar esta preferencia por la experiencia inicial no se desarrolla al incrementar el valor del intervalo, sino que tiende a la indiferencia entre ambas experiencias (Alvarado et al., 2006; López-Romero et al., 2010).

Estos resultados son coherentes con las predicciones de la RPT y apoyan la idea de que la RPT puede predecir adecuadamente el grado de recuperación de la respuesta que ha sido extinguida, en base al valor del IR y del valor subjetivo de cada experiencia. De esta forma, cuando la consecuencia de la primera experiencia tiene un valor subjetivo mayor, se presenta el efecto del cambio recencia-primacía conforme aumenta el valor del intervalo de entrenamiento-prueba y por tanto la RE observada será mayor. Tal y como ocurre en los procedimientos de adquisición-extinción (Ellson, 1938; Stout et al., 2005) Esta idea es sustentada adicionalmente con los resultados encontrados por Alvarado et al. (2006), en donde las consecuencias tuvieron un valor subjetivo similar, por lo que se observó una curva de recuperación plana, dando lugar a un cambio recencia-indiferencia. 
Los cambios recencia-primacía y recencia-indiferencia pueden ser adecuadamente predichos y explicados por la RPT. La siguiente ecuación reproduce la propuesta original de Devenport (1998) para la estimación temporal de una experiencia dada:

$$
\mathrm{VW}=\sum_{n=1}^{n=j}(\mathrm{Q} \times 1 / \mathrm{T}) / \sum_{n=1}^{n=j} 1 / \mathrm{T}
$$

En donde Vw corresponde al valor de estimación temporal de una experiencia, $Q$ a su valor subjetivo estimado, 1/T al valor de recencia. Así para calcular el valor de estimación temporal de la segunda experiencia $\mathrm{B}$ se debe de calcular $V w$ de $B$ considerando la estimación temporal total de ambas experiencias, es decir:

$$
\text { (2) } V w B=V w B / V w A+V w B
$$

Cuando los valores subjetivos de las experiencias $A$ y $B$ son iguales ( $Q A=$ QB) la función obtenida será de forma asintótica (positivamente acelerada) al disminuir la recencia de ambas experiencias con el paso del tiempo. Esto es, se preferirá inicialmente la experiencia más reciente $B$ y al paso del tiempo se tendera a la indiferencia entre ambas experiencias $A$ y $B$, tal y como ocurre en aquellos casos en los que el valor de ambas experiencias es igual y donde se ha observado una cambio recencia-indiferencia (Alvarado et al., 2006; LópezRomero et all.., 2010). Para el caso del presente experimento donde el valor de las experiencias es mayor para la experiencia $A$ (adquisición) que para la segunda experiencia $B$ de extinción ( $Q A>Q B)$. La función obtenida con el paso del tiempo tendera a la disminución de la estimación temporal de la experiencia $\mathrm{B}$ y a la recuperación de la experiencia $\mathrm{A}$ tal y como ocurre en el presente estudio y en estudios anteriores que han observado una cambio recencia-primacía (Ellson, 1938; Stout et al., 2005).

Los datos observados en este experimento sugieren que después de un entrenamiento sucesivo de dos fases y de un IR existe una integración de información de las memorias de ambas fases de entrenamiento que toma en cuenta el valor subjetivo y la distancia relativa de todas las experiencias de aprendizaje de acuerdo a lo propuesto por Devenport (1998). La RPT al tomar en cuenta estas variables se convierte en una herramienta que permite predecir adecuadamente la ocurrencia y desarrollo de la RE tanto en animales como en humanos.

De este manera mediante el estudio de los parámetros del valor subjetivo y de la recencia de cada experiencia, es posible conciliar datos aparentemente contradictorios como los son el cambio recencia-primacía observado por Stout et al. 
(2005) en aprendizaje causal. Con el cambio recencia-indiferencia observado por Alvarado et al. (2006) empleando una preparación similar.

Por otro lado, una diferencia entre la aproximación de la RPT y la explicación de la RE sustentada por Bouton (1993), estaría dada en que para la RPT el tiempo no es considerado como un cambio en el contexto físico. Sino que éste es considerado como una variable adicional que facilita la recuperación de información de manera similar a como lo hace un cambio de contexto físico, de ahí que ambas variables puedan interactuar y potenciar la recuperación de información (Rosas et al., 2001; Vila et al., 2002). Sin embargo en la RPT el efecto del paso del tiempo sobre la memoria no corresponde a un ajuste lineal sino logarítmico, ya que el efecto inicial de la recencia de una memoria se desvanece con el paso del tiempo para posteriormente igualarse con las memorias de experiencias pasadas. Es esta idea lo que hace que esta consideración teórica sea compatible con algunos puntos de vista actuales sobre tiempo y aprendizaje (Gallistel \& Gibbon, 2002).

Los presentes resultados cuestionan además las explicaciones tradicionales de la RE en base al efecto recencia-primacía (Miller, 2006; Pineño \& Miller, 2005) en las que se considera que la recuperación espontánea ocurrirá en una situación de interferencia entre claves o consecuencias en las que el paso del tiempo en algún momento llevará de manera abrupta a un cambio recenciaprimacía, debido a que las asociaciones aprendidas inicialmente son más fuertes.

Sin embargo, es necesario el estudio de un mayor número de predicciones de la RPT lo que nos permitirá a futuro contar con una herramienta con un mayor poder predictivo en el entendimiento de la recuperación espontánea.

\section{Referencias}

Alonso, L., Moreno, R., Vázquez, M., Del Rosal, E., \& Santacreu, J. (2005). Recuperación espontánea de la respuesta condicionada en un agente autónomo.Estudios de Psicología.Revista Trimestral de Psicología General, 26, 365-376.

Alvarado, A., Jara, E., Vila, J., \& Rosas, J. M. (2006). Time and order effects on causal learning. Learning and Motivation, 37, 324-345.

Bouton, M E. (1993). Context, time and memory retrieval in the interference paradigms of Pavlovian learning. Psychological Bulletin, 114, 80-99.

Devenport, L.D. (1998). Spontaneous recovery without interference: Why remembering is adaptive? Animal Learning \& Behavior, 26, 172-181.

Devenport, L. D., \& Devenport, J. A. (1994). Time-dependent averaging of foraging information in least chipmunks and golden-mantled ground squirrels. Animal Behaviour 47, 787-802. 
Ellson, D. G. (1938). Quantitative studies of the interaction of simple habits. I. Recovery from specific and generalized effects of extinction. Journal of Experimental Psychology, 23, 339-358.

Gallistel, Ch. R., \& Gibbon, J. (2002). The symbolic foundations of conditioned behavior. Erlbaum: Nueva Jersey.

Knoedler, A.J. Hellwig, K. A., \& Neath, I. (1999). The shift from recency to primacy with increasing delay. Journal of Experimental Psychology: Learning, Memory \& Cognition, 25, 474-487.

López-Romero, L. J., García-Barraza, R., \& Vila J. (en prensa). Spontaneous recovery does not appear as a recency-to-primacy shift as a function of the retention interval value in human instrumental learning. Behavioral Proccesses.

Miller, R. R. (2006). Challenges facing contemporary associative approaches to acquired behavior. Comparative Cognition \& behavior, 1, 77-93.

Pavlov, I. (1927). Conditioned Reflexes. Inglaterra: Oxford University Press.

Pineño, O., \& Miller, R. R. (2005). Primacy and recency effects in extinction and latent inhibition: A selective review with implications for models of learning. Behavioral Processes, 69, 223-235.

Postman, L., Stark, K., \& Fraser, J. (1968) Temporal changes in interference. Journal of Verbal Learning \& Verbal Behavior, 7, 672-694.

Romero, M., Vila, J., \& Overmier, B. (2007). Análisis de dos variables en la recuperación de información con humanos adultos jóvenes. Revista Colombiana de Psicología, 16, 31-48.

Rosas, J. M. Vila, J., Lugo, M., \& López, L. (2001). Combined effects of context change and retention interval on interference in causality judgments. Journalof Experimental Psychology: Animal Behavior Processes. 27, 153164.

Spear, N. E. (1973). Retrieval of memory in animals. Psychological Review, 80, 163-194.

Stollhoff, N., Menzel, R., \& Eisenhardt, D. (2005). Spontaneous recovery from extinction depends on the reconsolidation of the acquisition memory in an appetitive learning paradigm in the honeybee (Apis mellifera). The Journal of Neuroscience, 25, 4485-4492.

Thomas, B. L., Larsen, N., \& Ayres, J. J. B. (2003). Role of context similarity in $A B A, A B C$ and $A A B$ renewal paradigms: Implications for theories of renewal and for treating human phobias. Learning \& Motivation, 34, 410436.

Vila, N. J., Romero, M., \& Rosas, J. M. (2002). Retroactive interference after discrimination reversal decreases following temporal and physical context changes in human subjects. Behavioral Processes, 59, 47-54. 